Journal of Computer Science 2 (10): 789-793, 2006

ISSN 1549-3636

(C) 2006 Science Publications

\title{
Performance Analysis of Call Admission Control in WCDMA Systems with Mobility based Interference Margin Reservation
}

\author{
S. Malarkkan and V.C. Ravichandran \\ Department of ECE, College of Engineering, Anna University \\ Guindy, Chennai. Pin Code 600 025, India
}

\begin{abstract}
This study presents performance of call admission control and resource reservation schemes based on the mobility of the users in WCDMA cellular systems. In order to guarantee the handoff dropping probability, the mobility of the user is predicted based on a realistic mobility model. The mobility prediction scheme used in this study is used to estimate the set of candidate cells into which the mobile may move in the near future and calculates the likeliness value for each candidate cell. It also estimates the time slot at which the mobile may enter into the candidate cells based on the distance between the current location of the mobile and the candidate cell center distance. Based on the mobility prediction, resource is reserved in terms of Interference Guard margin (IGM) to guarantee some target handoff dropping probability. The admission threshold is adaptively controlled to achieve a better balance between guaranteeing handoff-dropping probability and maximizing resource utilization. Simulation results show that the call admission control scheme with mobility based resource reservation scheme outperforms well when compared with the fixed reservation scheme.
\end{abstract}

Key words: Blocking probability, dropping probability, most likely cell time

\section{INTRODUCTION}

The wide-band CDMA (W-CDMA) technology has emerged as the main air interface for $3 \mathrm{G}$ wireless systems, which promises to provide a transmission rate from $144 \mathrm{Kbps}$ to $2 \mathrm{Mbps}$, enabling multimedia services as those provided by broadband wired networks. The Radio Resource Management (RRM) module in the cellular network system is responsible for efficient utilizing of air interface resources and guarantees a certain QoS level to different users according to their traffic profiles. The call admission control (CAC) mechanism is one of the most important components of RRM. The radio resource-reservation estimation (RRE) mechanism helps CAC to decide the amount of resource to be reserved based on the mobility of the users in order to provide QoS guarantees to all users.

In this study, the performance of call admission control scheme with a dynamic resource reservation scheme in terms of Interference guard margin for multimedia traffic is analysed. The system resource is allocated efficiently by using dynamic resource reservation estimation (RRE) and CAC. The dynamic resource reservation estimator estimates the amount resource to be reserved in each and every cell so that the dropping probability can be minimised. However, this operation is challenged with two obstacles: determining where the subscriber will move to and the increase in call blocking. The set of candidate cells is estimated based on the mobility prediction and the reservation in some of the candidate cells may be redundant and will increase the call blocking rate unnecessarily. In order to reduce the amount of unnecessary reservations, a likeliness value with each reservation request is associated. Thus the amount of resource reserved in each cell is determined by the likeliness of all incoming reservation requests to that cell. The likeliness value of each candidate cell is determined by the mobility pattern of the mobile.

The mobility model used in the evaluation of a cellular system plays an important role in the validity of the evaluation. A realistic mobility model is used in this work to test the performance of the admission control scheme with resource reservation. Threshold priority policy (TPP) is introduced in ${ }^{[1]}$ against Cutoff Priority Policy(CPP).A new call is accepted in TPP only if the number of new calls is below a certain threshold. However, in CPP the total number of calls is limited. Reservations are implemented in terms of fixed number of guard channels and a very simple mobility model is used for the evaluation of the performance of the CAC scheme. $\mathrm{In}^{[2]} \mathrm{Ma}$ introduces a scheme with soft guard channels for CDMA systems by considering only the number of channels. However $\mathrm{Ma}^{[2]}$ does not consider the soft capacity and interference sensitivity of the CDMA systems and does not explain how reservation area is determined. Levin introduces shadow cluster concept $^{[3]}$ for TDMA/FDMA hybrid systems. In ${ }^{[4]}$, Shin proposes an interference based reservation scheme for CDMA systems. However the number of guard channels is fixed. Lie introduces in ${ }^{[5]}$ a similar scheme to the one in $^{[4]}$, considering signal-to-interference

Corresponding Author: S. Malarkkan, Research Scholar, Department of ECE, College of Engineering, Anna University, Guindy, Chennai. Pin Code 600 025, India 
ratio(SIR) instead of interference. $\mathrm{In}^{[6]}$, a dynamic channel reservation scheme is proposed in which only the new call and handoff arrival rates at a cell are considered. Useless reservation out off the moving direction of the mobile are made $\mathrm{in}^{[6]}$. $\mathrm{In}^{[7]}$, the subscribers are classified into high-speed and low-speed groups and an influence curve is drawn based on dwell time and subscriber speed.

Determination of reservation area: In order to avoid premature termination of the call without the intention of the users, channels must be reserved in the surrounding area. This part of the terrain is called the reservation area for the user. The construction of the reservation area is a two step process: finding the candidate cell in which a reservation is advised(but not required) and the calculation of the likeliness value for each reservation request.

Finding the candidate cells: Finding the set of candidate cells into which a user nay move depends on observing the following important points.

A user tends to keep his direction since he moves towards a destination, though there will be small changes in his direction due to obstacles in the terrain.

The obstacle in the terrain affects all individuals of a society in the same manner. i.e., if a high way has an arc to the left, all the vehicles draw an arc to the left.

The frequency of change in the direction signifies the type of structure the user is on. A user on the highway follows a path close to a straight line where a user on the streets often changes his direction.

The reservation area for a user is an elliptical region surrounding the mobile station. An ellipse can be defined by its size in both major and minor axes, the orientation and coordinates of one of its two foci. The orientation is the angle between the major axis of the ellipse and the $\mathrm{x}$-axis of the coordinate system. The size in the axes and orientation of the ellipse are determined by the mobility pattern of the user. Of the two foci of the ellipse, the first one is located at the current locus of the user and the ellipse is drawn such that the second focus is on the moving direction of the user. In other words, the direction of the user determines the orientation of the elliptical region.

The length in the major axis can be determined from the speed of the user and the length of the duration for which the system tries to make reservation. Higher speeds and longer reservation duration tend to increase the length in the major axis. The width in the minor axis can be determined from the length in the major axis and the mobility pattern of the user in the near past. Thus, the width of the ellipse depends implicitly on the speed and reservation duration in addition to the mobility pattern of the user.

The reservation area of the user who has been keeping its direction for some time will be a very narrow ellipse while the reservation area of a subscriber who has been changing its direction frequently will be wider. In the extreme case, the reservation area will be a circle.

Once the reservation area has been determined, the set of candidate cells can be determined by finding the cells intersected by the reservation area. In a hexagonal grid, this task can be accomplished by checking whether the center of the cell is inside a hypothetical ellipse, co-centered with the reservation area, but larger by $2 \mathrm{R}$ in length and width where $\mathrm{R}$ is the cell radius.

Calculation of the likeliness value: The likeliness that a subscriber will be at a specific point is related to the distance and the angle between the point and current position of the user. The distance and likeliness value are inversely proportional since the probability that the user changes his direction increases with distance.

A point on the moving direction of the user is more likely to be visited with respect to a point on the side. Therefore, the angle between the moving direction of the user and the line connecting the current location of the mobile and the point of concern is inversely proportional with the likeliness. The likeliness value can be calculated as:

$P i, j\left(T_{k}\right)=$ w1. [ $\left.1-\mathrm{d}_{\mathrm{j}} /(2 \cdot \mathrm{a}+\mathrm{R})\right]$

$+\mathrm{w} 2 .|\theta 1-\theta 2| / \pi$

(1)

where

$\mathrm{Pi}, \mathrm{j}\left(\mathrm{T}_{\mathrm{k}}\right)$ is the likeliness (probability)that the mobile in the cell ' $\mathrm{i}$ ' visit the cell in the time slot ' $\mathrm{T}_{\mathrm{k}}$ '.

$\mathrm{d}_{\mathrm{j}} \quad$ is the distance between the mobile and cell ' $\mathrm{j}$ '

w1 is the weight associated with the mobile- cell center distance.

w2 is the weight associated with the location of the cell center with respect to the direction of the mobile.

$\theta 1$ is the angle between the line that connects the mobile to the cell center and $\mathrm{x}$-axis.

$\theta 2$ is the direction of the mobile.

A is the semi-major axis( Half of the length) of the ellipse representing the reservation area.

The most likely cell-time(MLCT): When a mobile is active in cell $i$, we can get the Most Likely Cell-Time (MLCT) of that mobile, a cluster of time units at a cluster of cells when and where a mobile will most likely visit in the future. We select cells and time slots with $P i, j\left(T_{k}\right)$ greater than MLCT threshold, a design parameter, to form the MLCT of this mobile.

Capacity and load estimation in CDMA systems: The capacity of a CDMA system is limited by the total interference it can tolerate, which is called the interference-limited system. Users with different traffic profiles and attributes, such as the service rate, the signal-to-Interference ratio (SIR) requirement, media activity, etc., introduce different amount of interference to the system. The effect of interference increase for 
traffics with the following attributes for user ' $i$ ' is studied in literature.

$\mathrm{R}_{\mathrm{i}}$ : service (data) rate for user $\mathrm{i}$.

$\varepsilon_{\mathrm{i}}$ : target signal-to-interference ratio (SIR).

$v$ : media activity level

f: other cell to own cell interference ratio

To derive the amount of interference $\varepsilon_{i}$ introduced by user $i$ with data rate $R_{i}$ and target SIR, we have

$$
E_{i} \equiv\left(E_{\mathrm{b}} / N_{0}\right)_{i}=\frac{S_{i} /\left(\nu_{i} R_{i}\right)}{\left(I_{\text {total }}-S_{i}\right) / W},
$$

where $I_{\text {total }}$ is the total received power from $N$ active users at the base station, i.e.

$$
I_{\text {tetal }}=\sum_{i=1}^{N} S_{i}+P_{N}
$$

$E_{b}$ is the energy per user bit, $N_{0}$ the noise spectral density, $S_{i}$ and $v_{i}$ are the received power at the base station from user $i$ and the activity level of user $i$, respectively and $P_{N}$ is background noise. $I_{\text {total }}$ is limited by an upper-bound $I_{T h}$ for a system. When $I_{\text {total }}$ is higher than the upper-bound, the system is unstable and the overall interference increases dramatically. By rewriting (Eqn:2), we can express the received power $\mathrm{Si}$ for user $i$ at the base station as

$$
\Delta p_{i} \equiv\left(1+\frac{W}{\epsilon_{i} \cdot \nu_{i} \cdot R_{i}}\right)^{-1}
$$

where

$$
S_{i}=\left(1+\frac{W}{\epsilon_{i} \cdot \nu_{i} \cdot R_{i}}\right)^{-1} \cdot I_{\text {total }}=\Delta p_{i} \cdot I_{\text {total }}
$$

is called the load factor increment ${ }^{[8,9]}$. The current load factor of such an interference system is the sum of load factor increments brought by $N$ active mobile users, i.e.

$$
\rho=\sum_{i=1}^{N} \Delta \rho_{i}
$$

Shapira and Padovani ${ }^{[10]}$ and Holma et al. ${ }^{[8,9]}$ estimated the interference increase by taking into account the load curve as shown in Fig. 1. The ratio of $I_{\text {total }}$ to background noise $P_{N}$ is called noise-rise and denoted by $\eta$. The maximum value of $\eta$, denoted by $\eta_{\max }$, is normally set to $10^{[9]}$. The noise-rise $\eta$ is

$$
\eta \equiv \frac{I_{\text {total }}}{P_{N}}=\frac{\sum_{i=1}^{N} S_{i}+P_{N}}{P_{N}}=(1-\rho)^{-1}=\left(1-\sum_{i=1}^{N} \Delta \rho_{i}\right)^{-1} .
$$

By taking the partial derivative of $I_{\text {total }}$ with respect to $\rho i$, we get

$$
\frac{\Delta I_{i}}{\Delta \rho_{i}} \equiv \frac{\partial I_{\text {todat }}}{\partial \rho_{i}}=\frac{\partial\left(P_{N} /(1-\rho)\right)}{\partial \rho_{i}}=\frac{P_{N}}{(1-\rho)^{2}}=\frac{I_{\text {todal }}}{(1-\rho)} .
$$

The interference increment, $\Delta I i$ can be expressed in term of $R i$ as shown below:

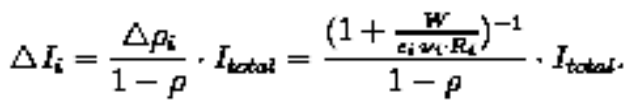

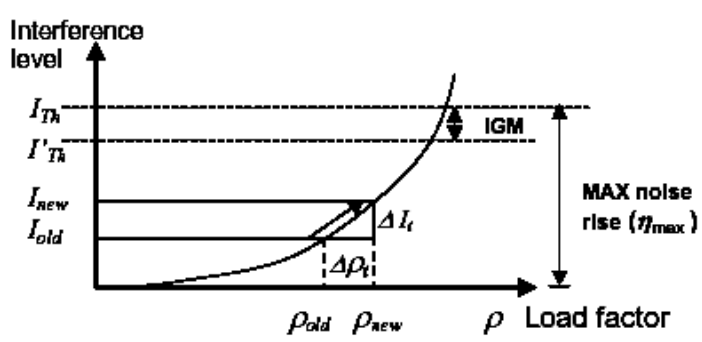

Fig. 1: The load curve and load estimation

The load curve serves as a good tool for interference increment estimation in our proposed scheme. The key point here is that given a data rate $R i$, the load increment $\Delta \rho_{i}$ can be computed to yield interference increment $\Delta I_{i}$.

Preferential treatment with IGM: In this section, an efficient radio resource management scheme based on the concept of interference guard margin (IGM) is discussed.

Service model: In a mobile communication system with $N$ active mobile users, the $i$ th $(i<N)$ user's traffic profile, which characterizes its services, is described as $\mathrm{S}(i)=\left\{r_{i},\left(R_{\max }, R_{\min }\right) i, \Pi_{i}, M_{i}\right\}$,

where $r_{i},\left(R_{\max }, R_{\min }\right) i, \Pi_{i}$ and $M_{i}$ in $\mathrm{S}(i)$, denote user $i$ 's rate adaptivity, service rate range, priority and mobility, respectively. The proposed service model is designed to take advantage of modern coding schemes and advanced mobile communication technologies as described below.

First, $r i$ is a binary indicator that indicates whether the user can be serviced at reduced bit-rates when the system is congested. Second, the service rate range $\left(R_{\text {max }}, R_{\text {min }}\right) i$ describes the target bandwidth consumption. If the network has enough resources, the request can be admitted at $R_{\max }$. If the cellular system is overloaded (congested), a rate-adaptive user can be serviced at a lower rate (down to $R_{\max } / 2$ or even $R_{\min }$ ) with degraded quality of service. Adaptation only takes place at the time of admitting new calls or at handoff epochs. Third, the priority tag $\Pi i$ helps the system to identify high priority users, who are likely to receive better QoS guarantees. Each different mobility traffic has a different weighting factor to estimate the amount of resources necessary to be reserved.

Interference guard margin: Interference Guard Margin (IGM) is a natural extension of the guard channel idea developed in the context of TDMA/FDMA systems by considering the load factor for system capacity estimation in CDMA systems. As illustrated in Fig. 1, we have the following two operations. First, the load curve is used to estimate the load increase as well as the interference increase. Second, a certain amount of IGM, instead of guard channels, is pre-reserved for high priority calls. The 791 
amount of IGM is dynamically adjusted by the RRE module.

For a new call to be admitted, the total interference level should not exceed the upper bound of the interference with threshold $I_{T h}$ that the system can tolerate. In addition to the constraint of $I_{T h}$, a lower priority call should comply with the augmented constraint $I_{T h}^{\prime}$. The margin between $I_{T h}$ and $I_{T h}^{\prime}$ is exactly the guard margin, which provides the preferential treatment to high priority calls by limiting the access to the low priority calls. The interference guard margin is given as,

$$
\operatorname{IGM}(j)=\alpha \sum_{i \in M L C T} P_{i, j}(T k) \cdot \Delta \mathrm{I}_{\mathrm{min}, \mathrm{i}}
$$

Call admission control (CAC) algorithm: The CAC algorithm for a media type with three scalable rates is discussed in this topic. In this, a new call or a handoff call can be admitted into the system with three data rates : $R_{\max }, R_{\text {half }}$ and $R_{\min }$. It can be generalized to a media type consisting of even more rates. Note that $I G M_{\text {new }}$ and $I G M_{\text {handoff }}$ are the estimated interference margin required to be reserved for new and handoff calls, respectively. The basic concept behind CAC is to test whether there is enough system resource left to serve the current call request at a certain rate, after reserving the necessary resource for preferential treatment. The CAC test is performed according to the following steps:

Step 1: New or handoff call test: An incoming call is first identified as a new or a handoff call type to decide its priority.

Step 2: Rate adaptivity test: The rate adaptivity of a new call (handoff call) is tested to decide whether it can be serviced at a lower data rate if the system is congested.

Step 3: Non-rate adaptive call test: If the call is rateadaptive, go to Step 4. Otherwise, test whether the amount of interference after admitting the current call and reserving the estimated IGM will exceed the maximum interference level that the system can tolerate.

Step 4: Rate adaptive call test: If the call is rateadaptive, the current call could be serviced at rates of $R_{\text {max, },}, R_{\text {half }, i}$ and $R_{\text {min, }, \text {, depending on }}$ the system traffic condition. The amount of interferences introduced by a call are $\Delta \mathrm{I}_{\max , \mathrm{i}}$, $\Delta \mathrm{I}_{\text {half,i }}$ and $\Delta \mathrm{I}_{\text {min,i }}$ when it is serviced at rates $\mathrm{R}_{\mathrm{max}, \mathrm{i}}, \mathrm{R}_{\text {half,i }}$ and $\mathrm{R}_{\min , \mathrm{i}}$ respectively. Then, we test the admission criteria by the order of data rates for the highest to the lowest. The call is served at its highest admissible rate.

\section{RESULTS}

A network topology with seven cells, which cover a region in a non-overlapped fashion, is considered in the simulation. The maximum interference level $I_{T h}$ is normally set to ten times of background noise, i.e. $\eta \max =10$. The same radio frequency band is reused for every cell and separated frequency bands are used for the reverse link and the forward link. The calls are generated from the mobile according to Poisson distribution with different mobility characteristics (equally distributed).The Poisson call arrival rate and the exponentially distributed call holding time are assumed.

Values used in the traffic profile $\mathrm{S}(i)$ of user $i$ are listed below:

i. $\quad r i \in$. $\{Y E S, N O\}$

ii. $\quad R_{\max , i}$ are, respectively, set to $19.2 \mathrm{Kbps}, 38.4 \mathrm{Kbps}$ and $76.8 \mathrm{Kbps}$ for voice, audio and video traffics and $R_{\min , i}$ is set to be $R_{\max , i} / 2$,

iii. $\Pi \in\{$ new, handoff $\}$,

iv. $M i \in\{H I G H, M O D, L O W\}$,

Communication system parameters used in simulation include: CDMA

chip rate $W=3.84 M c p s$, media activity $i=1$ and target SIR $\varepsilon i=7 d B$.

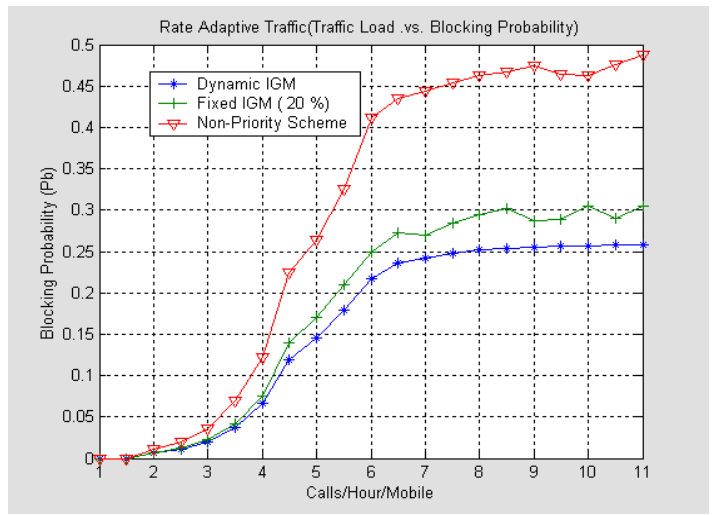

Fig. 2: Load vs. blocking probability-rate adaptive users

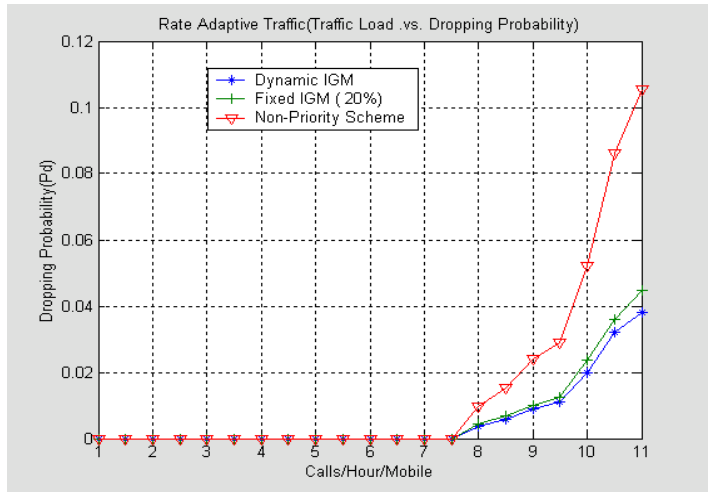

Fig. 3: Load vs. dropping probability-rate adaptive users 
The QoS performance of the call admission control schemes in terms of the blocking probability and dropping probability with the following four scenarios are compared.

* Non-priority scheme (also referred to as the complete sharing scheme),

* Fixed IGM $20 \%$ scheme (i.e. IGM is fixed to $20 \%$ $\mathrm{I}_{\mathrm{Th}}$, ,

* Dynamic IGM scheme with $\alpha=1$.

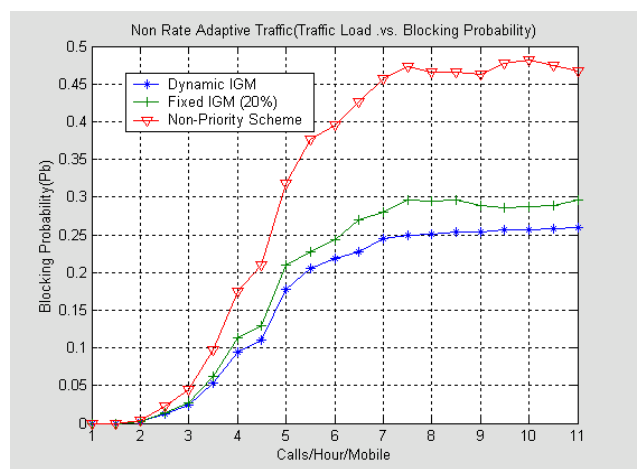

Fig. 4: Load vs. blocking probability-non rate adaptive users

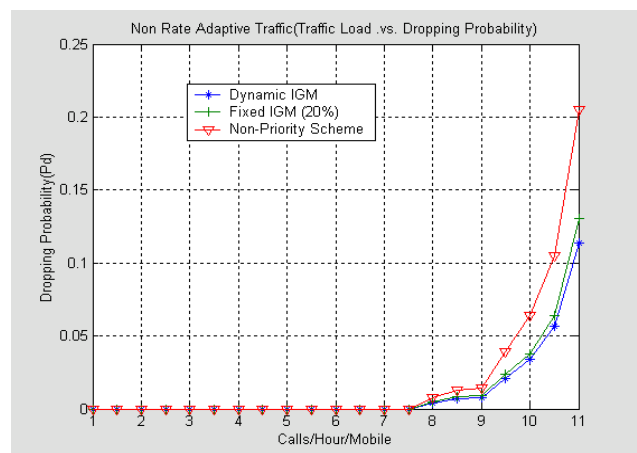

Fig. 5: Load vs. dropping probability-non rate adaptive users

The dynamic IGM scheme can adapt well to each traffic condition and has the best QoS performance. The new call blocking probability $P b$ and the handoff call dropping probability $P d$ with respect to traffic load are shown in the Fig. 2 and 3 for rate adaptive traffic and in Fig. 4 and 5 for non-rate adaptive traffic. Again, the dynamic IGM scheme has the best performance under the light as well as the moderate traffic loads. The performance is evaluated under the moderate to the heavy traffic loads with $\lambda$ varying from 1 to 5 (calls per hour per user). Results show that the dynamic IGM scheme significantly reduces the handoff dropping probability without much increase the new call blocking probability.

It is also noted that the dynamic IGM scheme outperforms the non-priority scheme for both rate adaptive and non-rate adaptive cases. Furthermore, the dynamic IGM scheme achieves a more significant improvement when the rate adaptive mechanism is available.

\section{CONCLUSION}

Effective radio resource management schemes, including dynamic radio resource estimation (RRE) and call admission control (CAC), based on the concept of interference guard margin (IGM) for Wideband CDMA systems were presented and its performance is analysed. A service model that included mobile terminals' service rate, their different levels of priority, rate adaptivity as well as their mobility is considered. The dynamic IGM scheme reserves a certain amount of interference margin for high priority handoff calls by referencing the traffic condition and mobile users' traffic profile in neighboring cells. The mobility-aware weighted sum plays an important role in the RRE process so that the effect of different mobility is taken into consideration. It was shown by computer simulation that the fixed and dynamic IGM schemes outperform the non-priority scheme in giving a smaller blocking and dropping probabilities under light as well as heavy traffic conditions.

\section{REFERENCES}

1. Gavish, B. and S. Sridhar, 1997. Threshold Priority policy for channel assignment in cellular networks. IEEE Trans. Computers, 46: 367-370.

2. Ma, Y., J.J. Han and K.S. Trivedi, 2000. Call admission control for reducing dropped calls in CDMA cellular systems. IEEE Infocom, pp: 14811490.

3. Levine, D.A., I.F. Akyildiz and M. Naghshineh, 1997. A resource estimation and call admission algorithm for wireless multimedia networks using shadow cluster concept, Trans. Networking, 5: 112.

4. Shin, S.M., C.H. Cho and D.K. Sung, 1999. Interference based channel assignment for DSCDMA cellular systems. IEEE Trans. Vehicular Technol., 48: 1.

5. Liu, Z. and M.E. Zarki, 1994. SIR- based Call admission control for DS-CDMA cellular systems. JSAC, 12: 638-644.

6. Kim, Y., C.D.E. Lee, B.J. Lee, Y.S. Kim and B. Mukherjee, 1999. Dynamic channel reservation based on mobility in wireless ATM networks. IEEE Commun. Mag., pp: 47-51.

7. Hou, J., Y. Fang and A.N. Akansu, 2000. Channel reservation based on mobility information in wireless networks. WCNC.

8. Holma, H. and J. Laakso, 1999. Uplink admission control and soft capacity with mud in CDMA. Proc. VTC'99 Fall, (Amsterdam, Netherlands), pp: 19-22.

9. Holma, H. and A. Toskala, 2000. WCDMA for UMTS, Radio Access For Third Generation Mobile Communications. John Wiley \& Sons.

10. Shapira, J. and R. Padovani, 1994. Microcell engineering in cdma cellular networks. IEEE Trans. Vehicular Technol., 43: 213-216. 